\title{
Markov Functional Modeling of Equity, Commodity and other Assets
}

\author{
Christian P. Fries \\ email@christian-fries.de
}

March 31, 2006

(Version 0.8.3)

\section{Abstract}

In this short note we show how to setup a one dimensional single asset model, e.g. equity model, which calibrates to a full (two dimensional) implied volatility surface. We show that the efficient calibration procedure used in LIBOR Markov functional models may be applied here too. In a addition to the calibration to a full volatility surface the model allows the calibration of the joint asset-interest rate movement (i.e. local interest rates) and forward volatility. The latter allows the calibration of compound or Bermudan options.

The Markov functional modeling approach consists of a Markovian driver process $x$ and a mapping functional representing the asset states $S(t)$ as a function of $x(t)$. It was originally developed in the context of interest rate models, see [7]. Our approach however is similar to the setup of the hybrid Markov functional model in spot measure, as considered in [5].

For equity models it is common to use a deterministic Numéraire, e.g. the bank account with deterministic interest rates. In our approach we will choose the asset itself as Numéraire. This is a subtle, but crucial difference to other approaches considering Markov functional modeling. Choosing the asset itself as Numéraire will allow for a very efficient numerically calibration procedure. As a consequence interest rates have to be allowed to be stochastic, namely as a functional of $x$ too. The Black-Scholes model with deterministic interest rates is a special case of such a Markov functional model.

The most general form of this modeling approach will allow for a simultaneous calibration to a full two dimensional volatility smile, a prescribed joint movement of interest rates and a given forward volatility structure. 


\section{Contents}

1 Introduction $\quad 3$

2 Markov Functional Model with Asset Numéraire 3

2.1 Markov Functional Assumption . . . . . . . . . . . . . . . . 3

3 Example: The Black-Scholes Model 4

4 Numerical Calibration to a Full Two Dimensional European Option Smile Surface 5

4.1 Market Price . . . . . . . . . . . . . . . . . . . . 5

4.2 Model Price . . . . . . . . . . . . . . . . . . . . . . . 5

4.3 Solving for the Functional . . . . . . . . . . . . . . 6

5 Interest Rates $\quad 6$

5.1 A Note on Interest Rates and the No-Arbitrage Requirement . . . . . . . . . . 6

5.2 Where are the Interest Rates? . . . . . . . . . . . . . . . . . . . . 6

6 Model Dynamics $\quad 7$

6.1 Introduction . . . . . . . . . . . . . . . . . 7

6.1 .1 Time Copula . . . . . . . . . . . . . . . . . . 8

6.1 .2 Time-Discrete Markovian Driver . . . . . . . . . . . . . . . . . 8

6.2 Interest Rate Dynamics . . . . . . . . . . . . . . . . . . . . 8

6.2.1 Example: Black-Scholes Model with a Term Structure of Volatility . . 8

6.2.2 Calibration to arbitrary Interest Rate Dynamics . . . . . . . . . . . . . 10

6.3 Forward Volatility . . . . . . . . . . . . . . . . . . . . 10

6.3.1 Example: Black-Scholes Model with a Term Structure of Volatility . . 10

6.3.2 Example: Exponential Decaying Instantaneous Volatility . . . . . . . . 11

7 Implementation $\quad 11$

7.1 Calibration of the Functional Forms . . . . . . . . . . . . . . 11

8 Conclusion $\quad 13$

$\begin{array}{lr}\text { List of Symbols } & 14\end{array}$

$\begin{array}{ll}\text { References } & 15\end{array}$

$\begin{array}{ll}\text { Notes } & 15\end{array}$ 


\section{Introduction}

Markov functional models are well known in interest rate [7] and interest rate hybrid modeling [5]. The main idea of Markov functional modeling is

- The asset to model is a function of an underlying low dimensional Markov process $x$.

- The model is such that the functional values can be determined from market prices by a simple inversion (e.g. through one dimensional root finders).

The first requirement allows for an efficient implementation on a low dimensional lattice. While one usually has to use Monte-Carlo methods for high dimensional models, low dimensional lattice methods show much better stability and performance.

The second requirement allows for a fast calibration that captures a continuum of market prices. Thus, one main feature of Markov functional models is their ability to calibrate perfectly to a given two dimensional smile surface, parametrized by maturity and strike.

In this short note we show how to setup a one dimensional single asset model, e.g. equity model, which calibrates to a full (two dimensional) implied volatility surface. We show that the efficient calibration procedure used in LIBOR Markov functional models, may be applied.

There have been similar attempts to this, e.g. [2, 8]. The novelty of our approach is that, in contrast to the common choice of a risk less bank account Numéraire, we choose the asset itself as Numéraire. This is a subtle, but crucial difference. Choosing the asset itself as Numéraire will allow for a very efficient numerically calibration procedure. As a consequence interest rates have to be allowed to be stochastic, namely as a functional of $x$ too. The Black-Scholes model with deterministic interest rates is a special case of such a Markov functional model.

Carr, Thalela and Zariphopoulou [3] showed that a functional model which chooses a risk less bank account Numéraire with deterministic interest rate may calibrate only to a one dimensional sub-manifold, namely the smile curve for a single maturity $T$ and a maturity curve of prices for a given strike. Conversely, specifying a functional model that calibrates to a full two dimensional implied volatility surface and simultaneously prescribing interest rate dynamics (e.g. as being deterministic) will result in a violation of arbitrage, [2]. This will come clear in our presentation from the Bond pricing formula (2).

\section{Markov Functional Model with Asset Numraire}

Consider a simple one dimensional Markov process, e.g.

$$
\mathrm{d} x(t)=\sigma(t) \cdot \mathrm{d} W^{\mathbb{Q}}(t), \quad x(0)=x_{0},
$$

where $\sigma$ is a deterministic function and $W^{\mathbb{Q}}$ denotes a $\mathbb{Q}$-Brownian motion. Without loss of generality we may assume $x_{0}=0$. Equation (1) is the most simplest choice of a Markovian driver process. We will consider the addition of a drift term to (1) in our discussion on model dynamics in Section 6.

Let $S(t)$ denote the time $t$ value of some asset for which we assume to have a continuum of European option prices. Let $x$ and $S$ be adapted stochastic processes defined on $\left(\Omega, \mathbb{Q}, \mathcal{F}_{t}\right)$, where $\left\{\mathcal{F}_{t}\right\}$ denotes the filtration generated by $W^{\mathbb{Q}}$.

\subsection{Markov Functional Assumption}

We assume that the time $t$ value of the asset $S$ is a function of $x(t)$, i.e. we assume the existence of a functional $(t, \xi) \mapsto S(t, \xi)$ such that

$$
S(t, \omega)=S(t, x(t, \omega)),
$$


where the left hand side denotes our asset value at time $t$ on path $\omega$ and the right hand side denotes some functional of our Markovian driver $x$, which we ambiguously name $S$. We allow for some ambiguity in notation here. From here on $S$ will also denote a deterministic mapping (the functional)

$$
(t, \xi) \mapsto S(t, \xi) .
$$

It will be clear from the arguments of $S$ if we speak of the functional $(t, \xi) \mapsto S(t, \xi)$ or of the process $t \mapsto S(t)$.

For $t_{1}<t_{2}$ we trivially have that

$$
\frac{S\left(t_{1}\right)}{S\left(t_{1}\right)}=\mathrm{E}^{\mathbb{Q}}\left(\frac{S\left(t_{2}\right)}{S\left(t_{2}\right)} \mid \mathcal{F}_{t_{1}}\right) .
$$

We now postulate that $\mathbb{Q}$ is the equivalent martingale measure with respect to the Numéraire $S$ and that a universal pricing theorem holds for all other traded products, i.e. that their $S$ relative price is a $\mathbb{Q}$-martingale.

This implies that the zero coupon bond $P(T ; t)$ having maturity $T$ and being observed in $t<T$ fulfills

$$
\frac{P(T ; t)}{S(t)}=\mathrm{E}^{\mathbb{Q}}\left(\frac{1}{S(T)} \mid \mathcal{F}_{t}\right)
$$

Using the functional representation of $S$ we find that $P(T ; t)$ is represented as a functional of $x(t)$ too, namely

$$
(t, \xi) \mapsto P(T ; t)
$$

with

$$
\frac{P(T ; t, \xi)}{S(t, \xi)}=\mathrm{E}^{\mathbb{Q}}\left(\frac{1}{S(T, x(T))} \mid\{x(t)=\xi\}\right) .
$$

\section{Example: The Black-Scholes Model}

Let us assume a Markovian driver with constant instantaneous volatility $\sigma(t)=\sigma$. For the Black-Scholes Model we have

$$
S(t, \xi)=S(0) \cdot \exp \left(r \cdot t+\frac{1}{2} \sigma_{\mathrm{BS}}^{2} t+\frac{\sigma_{\mathrm{BS}}}{\sigma} \cdot \xi\right),
$$

where $\sigma_{\mathrm{BS}}$ denotes the (constant) Black-Scholes volatility. Plugging this into (2) we find

$$
P(T ; t, \xi)=\exp (-r(T-t))
$$

so interest rates are indeed deterministic here.

This is indeed the Black-Scholes model: From the definition of the Markovian driver we have that $\frac{1}{\sigma} \cdot x(t)=W(t)$ and thus

$$
S(t, x(t))=S(0) \cdot \exp \left(r \cdot t+\frac{1}{2} \sigma_{\mathrm{BS}}^{2} t+\sigma_{\mathrm{BS}} \cdot W(t)\right) .
$$

In other words the $\mathbb{Q}$ dynamics of $S$ is ${ }^{1}$

$$
\mathrm{d} S(t)=r S(t) \mathrm{d} t+\sigma_{\mathrm{BS}}^{2} S(t) \mathrm{d} t+\sigma_{\mathrm{BS}} S(t) \cdot \mathrm{d} W^{\mathbb{Q}}(t) .
$$

Introducing a new Numéraire

$$
\mathrm{d} B(t)=r B(t) \mathrm{d} t, \quad B(0)=1
$$

\footnotetext{
${ }^{1}$ Note that $\mathbb{Q}$ is the equivalent martingale measure with respect to the Numéraire $S$.
} 
we find for the change of Numéraire process $\frac{S}{B}$ that

$$
\mathrm{d} \frac{S}{B}=\sigma_{\mathrm{BS}}^{2} S(t) \mathrm{d} t+\sigma_{\mathrm{BS}} S(t) \cdot \mathrm{d} W^{\mathbb{Q}}(t) .
$$

For $\frac{S}{B}$ to be a martingale under $\mathbb{Q}^{B}$ we have $\mathrm{d} W^{\mathbb{Q}}(t)=\mathrm{d} W^{\mathbb{Q}^{\mathbb{B}}}-\sigma_{\mathrm{BS}}^{2} \mathrm{~d} t$ and thus we have

$$
\begin{aligned}
\mathrm{d} S(t) & =r S(t) \mathrm{d} t+\sigma_{\mathrm{BS}} \cdot S(t) \cdot \mathrm{d} W^{\mathbb{Q}^{\mathbb{B}}}(t) \\
\mathrm{d} B(t) & =r B(t) \mathrm{d} t .
\end{aligned}
$$

Note: $\mathrm{d} W^{\mathbb{Q}}(t)$ is a $\mathbb{Q}$ Brownian motion, where $\mathbb{Q}$ is the equivalent martingale measure with respect to the Numéraire $S$, while $\mathrm{d} W^{\mathbb{Q}^{B}}(t)$ is a $\mathbb{Q}^{B}$ Brownian motion, where $\mathbb{Q}^{B}$ is the equivalent martingale measure with respect to the Numéraire $B$.

\section{Numerical Calibration to a Full Two Dimensional Euro- pean Option Smile Surface}

As for the interest rate Markov functional model we are able to calculate the functionals numerically from a given two dimensional smile surface. Our approach here is similar the approach for the one-dimensional LIBOR Markov functional model under spot measure, [5]. Consider the following time $T$ payout:

$$
V(T, K ; T):= \begin{cases}S(T) & \text { if } S(T)>K \\ 0 & \text { else. }\end{cases}
$$

Obviously we have

$$
V(T, K ; T)=\max (S(T)-K, 0)+K \cdot \begin{cases}1 & \text { if } S(T)>K \\ 0 & \text { else }\end{cases}
$$

i.e. the value of $V$ is given by the value of a portfolio of one call option and $K$ digital options, all having strike $K$. This is our calibration product.

\subsection{Market Price}

Let $\bar{\sigma}_{\mathrm{BS}}(T, K)$ denote the Black-Scholes implied volatility surface given from market prices. Then we have that the market price of $V$ is

$$
\begin{aligned}
& V^{\text {market }}(T, K ; 0) \\
& =\underbrace{S(0) \Phi\left(d_{+}\right)-\exp (-r T) K \Phi\left(d_{-}\right)}_{\text {call option part }}+K \underbrace{\exp (-r T)\left(\Phi\left(d_{-}\right)+S(0) \sqrt{T} \Phi^{\prime}\left(d_{+}\right) \frac{\partial \bar{\sigma}_{\mathrm{BS}}(T, K)}{\partial K}\right)}_{\text {digital part }} \\
& =S(0) \Phi\left(d_{+}\right)+K S(0) \sqrt{T} \Phi^{\prime}\left(d_{+}\right) \frac{\partial \bar{\sigma}_{\mathrm{BS}}(T, K)}{\partial K},
\end{aligned}
$$

where $\Phi(x):=\frac{1}{\sqrt{2 \pi}} \int_{-\infty}^{x} \exp \left(-\frac{y^{2}}{2}\right) \mathrm{d} y$ and $d_{ \pm}=\frac{\ln \left(\frac{\exp (r T) S(0)}{K}\right) \pm \frac{1}{2} \bar{\sigma}_{\mathrm{BS}}^{2}(T, K) T}{\bar{\sigma}_{\mathrm{BS}}(T, K) \sqrt{T}}$.

\subsection{Model Price}

Within our model the price of the product (4) is

$$
\begin{aligned}
V^{\text {model }}(T, K ; 0) & =S(0) \cdot \mathrm{E}^{\mathbb{Q}}\left(\frac{S(T, x(T)) \cdot \mathbf{1}_{\{S(T, x(T))>K\}}}{S(T)} \mid\left\{x(0)=x_{0}\right\}\right) \\
& =S(0) \cdot \mathrm{E}^{\mathbb{Q}}\left(\mathbf{1}_{\{S(T, x(T))>K\}} \mid\left\{x(0)=x_{0}\right\}\right)
\end{aligned}
$$


Assuming that our functional $(T, \xi) \mapsto S(T, \xi)$ is monotone increasing in $\xi$ we may write

$$
V^{\text {model }}(T, K ; 0)=S(0) \cdot \mathrm{E}^{\mathbb{Q}}\left(\mathbf{1}_{\left\{x(T)>x^{*}\right\}} \mid\left\{x(0)=x_{0}\right\}\right),
$$

where $x^{*}$ is the (unique) solution of $S\left(T, x^{*}\right)=K$. Note that (5) depends on $x^{*}$ and the probability distribution of $x(T)$ only and that $x(T)$ is known due to the simple form of our Markovian driver. It does not depend on the functional $S$ ! Thus for given $x^{*}$ we may calculate

$$
V^{\text {model }}\left(T, x^{*} ; 0\right):=S(0) \cdot \mathrm{E}^{\mathbb{Q}}\left(\mathbf{1}_{\left\{x(T)>x^{*}\right\}} \mid\left\{x(0)=x_{0}\right\}\right) .
$$

\subsection{Solving for the Functional}

For given $x^{*}$ we now solve the equation

$$
V^{\text {market }}\left(T, K^{*} ; 0\right)=V^{\text {model }}\left(T, x^{*} ; 0\right)
$$

to find $S\left(T, x^{*}\right)=K^{*}$ and thus the functional form $(T, \xi) \mapsto S(T, \xi)$. This can be done very efficiently using fast one dimensional root finders, e.g. Newton's method.

\section{Interest Rates}

\subsection{A Note on Interest Rates and the No-Arbitrage Requirement}

Functional models for equity option pricing have been investigated before, see e.g. [2] and [8]. However, their approach chooses deterministic interest rates and chooses the bank account as Numéraire. As suggested in Section 3 this will impose a very strong self similarity requirement on the functionals (which is fulfilled by the Black-Scholes model). Such models may calibrate only to a one dimensional submanifold of a given implied volatility surface, see [3]. For the Markov functional model this follows directly from (2). Assuming that the Markovian driver $x$ is given and that the interest rate dynamic $P(T ; t, \xi)$ is given, we find from (2) that

$$
S(t, \xi)=\frac{P(T ; t, \xi)}{\mathrm{E}^{\mathbb{Q}}\left(\frac{1}{S(T, x(T))} \mid\{x(t)=\xi\}\right)} .
$$

So once a terminal time $T$ functional $\xi \mapsto S(T, \xi)$ has been defined, all other functionals are implied by the interest rate dynamics $P$ and the dynamics of the Markovian driver.

Sticking to prescribed interest rates, the only way to allow for more general functional is to violate the no-arbitrage requirement (2) or change the Markovian driver. The latter will be considered in Section 6.

\subsection{Where are the Interest Rates?}

Our model calibrates to a continuum of options on $S$. We do not even specify interest rates. This is not necessary, since the specification of the interest rates is already contained in the specification of a continuum of options on $S$. Consider options on $S(T)$, i.e. options with maturity $T$. First note that from a continuum $K \mapsto V_{\text {call }}^{\text {market }}(T, K ; 0)$ of market prices for call option payouts

$$
V_{\text {call }}^{\text {market }}(T, K ; T)=\max (S(T)-K, 0)
$$

we obtain prices for the corresponding digital payouts

$$
V_{\text {digital }}^{\text {market }}(T, K ; T)= \begin{cases}1 & S(T)>K \\ 0 & \text { else }\end{cases}
$$


by

$$
V_{\text {digital }}^{\text {market }}(T, K ; 0)=-\frac{\partial}{\partial K} V_{\text {call }}^{\text {market }}(T, K ; 0) .
$$

Thus the value of the zero coupon bond with maturity $T$ is

$$
P(T ; 0)=\lim _{K \searrow 0} V_{\text {digital }}^{\text {market }}(T, K ; 0)=-\lim _{K \searrow 0} \frac{\partial}{\partial K} V_{\text {call }}^{\text {market }}(T, K ; 0) .
$$

Note that this argument is model independent.

Within the functional model, Equation (5.2) hold locally in each state. Given we are at time $t$ in state $x(t)=\xi$ we have for the corresponding bond that

$$
P(T ; t, \xi)=\lim _{K \searrow 0} V_{\text {digital }}^{\text {model }}(T, K ; t, \xi) .
$$

From this it is clear why specifying interest rates would represent a violation of the no-arbitrage requirement. This is precisely the reason why the models in [2, 8] allow for arbitrage.

In the next section we show that the model implied interest rate dynamics will likely be undesirable. However, as it is known from interest rate hybrid Markov functional models [5], it is possible to calibrate to different model dynamics by changing the Markovian driver $x$.

\section{Model Dynamics}

\subsection{Introduction}

Markov functional models calibrate perfectly to a continuum of option prices, i.e. to the market implied probability density of the underlying, cf. [1]. Indeed, the functional $(t, \xi) \mapsto S(t, \xi)$ is nothing more than the measure transformation from the probability density of $x(t)$ to the market implied probability density of the underlying $S(t)$.

While the calibration to terminal probability densities is a desirable feature, it is not the only requirement on a model, specifically if the model is used to price complex derivatives like Bermudan options. Here the transition probabilities play a role, i.e. the model dynamics. The most prominent aspects of model dynamics are

- Interest Rate Dynamics: For an equity Markov functional model the joint movement of the interest rate and the asset has to be analysed. It is possible to calibrate to a given interest rate dynamics by adding a drift to the Markovian driver, see Section 6.2.

- Forward volatility: This is the implied volatility of an option with maturity $T$ and strike $K$, given we are in state $(t, \xi)$, i.e.

$$
S(t, \xi) \cdot \mathrm{E}^{\mathbb{Q}}\left(\frac{\max (S(T, x(T))-K, 0)}{S(T)} \mid\{x(t)=\xi\}\right) .
$$

Obviously it will play an important role for compound options and Bermudan options. The forward volatility may be calibrated by changing the instantaneous volatility of the Markovian driver, see Section 6.3.

- Auto correlation / Forward spread volatility: The auto correlation of the process $S$ impacts the forward spread volatility. This is the implied volatility of an option on $S\left(T_{2}\right)-S\left(T_{1}\right)$ with maturity $T_{2}$, given we are in state $(t, \xi)$, i.e.

$$
S(t, \xi) \cdot \mathrm{E}^{\mathbb{Q}}\left(\frac{\max \left(S\left(T_{2}, x\left(T_{2}\right)\right)-S\left(T_{1}, x\left(T_{1}\right)\right), 0\right)}{S\left(T_{2}, x\left(T_{2}\right)\right)} \mid\{x(t)=\xi\}\right) .
$$


Markov functional models allow a limited calibration to different model dynamics by changing the dynamics of the Markovian driver $x$. For our choice

$$
\mathrm{d} x=\sigma(t) \cdot \mathrm{d} W(t)
$$

we may change the auto correlation of $x$ by choosing different instantaneous volatility functions $\sigma$. Since the calibration of the functionals is scale invariant with respect to the terminal standard deviation $\bar{\sigma}(t)$ of $x(t)$, the calibration to the terminal probability densities is independent of the choice of $\sigma$. See the Black-Scholes example in Section 3 for an example of this invariance.

\subsubsection{Time Copula}

The specification of the auto correlation of $x$ (through $\sigma$ ) is sometime called time copula, [2], since it may be specified through the joint distribution of $\left(x\left(t_{1}\right), x\left(t_{2}\right)\right)$. For this reasons similar functional models are sometimes called copula models, a term that is more associated with credit models, where joint default distribution is constructed from marginal default distributions.

In addition to a specification of the instantaneous volatility, the Markovian driver may be endowed with a drift.

\subsubsection{Time-Discrete Markovian Driver}

We assume a given time discretization $\left\{0=t_{0}<t_{1}<t_{2}<\ldots\right\}$ and consider the realizations $x\left(t_{i}\right)$ of the Markovian driver $x$ given trough increments $\Delta x\left(t_{i}\right)=x\left(t_{i+1}\right)-x\left(t_{i}\right)$. It is natural that a practical implementation of the model will feature a certain time discretization. Thus, speaking of calibration of a specific time discretized implementation, it is best to consider the Markovian driver given through an Euler scheme (as in (6), (7))

$$
x\left(t_{i+1}\right)=x\left(t_{i}\right)+\mu\left(t_{i}, x\left(t_{i}\right)\right) \Delta t_{i}+\sigma\left(t_{i}, x\left(t_{i}\right)\right) \Delta W\left(t_{i}\right) .
$$

\subsection{Interest Rate Dynamics}

\subsubsection{Example: Black-Scholes Model with a Term Structure of Volatility}

Let us first assume that the Markovian driver is given by

$$
x\left(t_{i+1}\right)=x\left(t_{i}\right)+\sigma_{i} \Delta W\left(t_{i}\right) .
$$

Consider a term structure of Black-Scholes implied volatilities, i.e. let $\bar{\sigma}_{\mathrm{BS}}\left(t_{i}\right)$ denote the implied volatility of an option with maturity $t_{i}$. Assuming the simple Markovian driver (6) the corresponding functionals that calibrate to these options are

$$
S\left(t_{i}, \xi\right)=S(0) \cdot \exp \left(r \cdot t_{i}+\frac{1}{2} \bar{\sigma}_{\mathrm{BS}}\left(t_{i}\right)^{2} t+\frac{\bar{\sigma}_{\mathrm{BS}}\left(t_{i}\right)}{\bar{\sigma}_{i}} \cdot \xi\right),
$$

where $\bar{\sigma}_{i}^{2}:=\frac{1}{t_{i}} \sum_{j=0}^{i-1} \sigma_{j}^{2} \Delta t_{j}$.

Already within this model a stochastic interest rate dynamic is implied. From (2) we find

$$
\begin{aligned}
P\left(t_{i+1} ; t_{i}, \xi\right)=S\left(t_{i}, \xi\right) \cdot \mathrm{E}^{\mathbb{Q}}\left(\frac{1}{S\left(t_{i+1}, x\left(t_{i+1}\right)\right)} \mid\left\{x\left(t_{i}\right)=\xi\right\}\right) \\
=\exp \left(-r \cdot\left(t_{i+1}-t_{i}\right)-\frac{1}{2}\left(\bar{\sigma}_{\mathrm{BS}}\left(t_{i+1}\right)^{2} \cdot\left(t_{i+1}-\frac{\sigma_{i}^{2}}{\bar{\sigma}_{i+1}^{2}} \Delta t_{i}\right)-\bar{\sigma}_{\mathrm{BS}}\left(t_{i}\right)^{2} \cdot t_{i}\right)\right. \\
\left.-\left(\frac{\bar{\sigma}_{\mathrm{BS}}\left(t_{i+1}\right)}{\bar{\sigma}_{i+1}}-\frac{\bar{\sigma}_{\mathrm{BS}}\left(t_{i}\right)}{\bar{\sigma}_{i}}\right) \cdot \xi\right),
\end{aligned}
$$


If the volatility of the Markovian driver $x$ decays faster than the implied Black-Scholes volatility, then interest rate will move positively correlated with the stock. If the volatility of the Markovian driver $x$ decays slower than the implied Black-Scholes volatility, then interest rate will move negatively correlated. ${ }^{2}$ If we choose the instantaneous volatility of $x$ such that $\bar{\sigma}_{i}=\bar{\sigma}_{\mathrm{BS}}\left(t_{i}\right)$ we have

$$
P\left(t_{i+1} ; t_{i}, \xi\right)=\exp \left(-r \cdot\left(t_{i+1}-t_{i}\right)\right)
$$

i.e. we recovered a model with deterministic interest rates. Reconsidering the case of a continuous driver $\mathrm{d} x=\sigma(t) \mathrm{d} W(t)$, we see that for $\frac{1}{t} \int_{0}^{t} \sigma(\tau)^{2} \mathrm{~d} \tau=\bar{\sigma}_{\mathrm{BS}}(t)^{2}$ the functionals above define a Black-Scholes model with instantaneous volatility $\sigma$.

However, we do not need to sacrifice the instantaneous volatility of $x$ to match the interest rate dynamics. A much more natural choice is to add a suitable drift to the Markovian driver $x$. Consider a Markovian driver $x$ such that

$$
x\left(t_{i+1}\right)=x\left(t_{i}\right)+\alpha_{i} x\left(t_{i}\right) \Delta t_{i}+\sigma_{i} \Delta W\left(t_{i}\right), \quad x\left(t_{0}\right)=x_{0}=0 .
$$

Note that the $x\left(t_{i}\right)$ 's are normal distributed with mean 0 (assuming $x_{0}=0$ ) and standard deviations $\gamma_{i} \sqrt{t_{i}}$ where

$$
\gamma_{i+1}^{2} t_{i+1}=\gamma_{i}^{2} t_{i} \cdot\left(1+\alpha_{i} \Delta t_{i}\right)+\sigma_{i}^{2} \Delta t_{i}
$$

Together with the the functionals

$$
\begin{aligned}
S\left(t_{i}, \xi\right) & =S(0) \cdot \exp \left(r \cdot t_{i}+\frac{1}{2} \bar{\sigma}_{\mathrm{BS}}\left(t_{i}\right)^{2}+\frac{\bar{\sigma}_{\mathrm{BS}}\left(t_{i}\right)}{\gamma_{i}} \cdot \xi\right) \\
S\left(t_{i+1}, \xi\right) & =S(0) \cdot \exp \left(r \cdot t_{i+1}+\frac{1}{2} \bar{\sigma}_{\mathrm{BS}}\left(t_{i+1}\right)^{2}+\frac{\bar{\sigma}_{\mathrm{BS}}\left(t_{i+1}\right)}{\gamma_{i+1}} \cdot \xi\right)
\end{aligned}
$$

We have

$$
\begin{aligned}
P\left(t_{i+1} ; t_{i}, \xi\right)= & S\left(t_{i}, \xi\right) \cdot \mathrm{E}^{\mathbb{Q}}\left(\frac{1}{S\left(t_{i+1}, x\left(t_{i+1}\right)\right)} \mid\left\{x\left(t_{i}\right)=\xi\right\}\right) \\
= & \exp \left(-r \cdot \Delta t_{i}-\frac{1}{2}\left(\bar{\sigma}_{\mathrm{BS}}\left(t_{i+1}\right)^{2} \cdot\left(t_{i+1}-\frac{\sigma_{i}^{2}}{\gamma_{i+1}^{2}} \Delta t_{i}\right)-\bar{\sigma}_{\mathrm{BS}}\left(t_{i}\right)^{2} \cdot t_{i}\right)\right. \\
& \left.-\left(\frac{\bar{\sigma}_{\mathrm{BS}}\left(t_{i+1}\right)}{\gamma_{i+1}}\left(1+\alpha_{i} \Delta t_{i}\right)-\frac{\bar{\sigma}_{\mathrm{BS}}\left(t_{i}\right)}{\gamma_{i}}\right) \cdot \xi\right) \\
= & \exp \left(-r \cdot \Delta t_{i}-\frac{1}{2}\left(\bar{\sigma}_{\mathrm{BS}}\left(t_{i+1}\right)^{2} \cdot \frac{\gamma_{i}^{2}}{\gamma_{i+1}^{2}}\left(1+\alpha_{i} \Delta t_{i}\right)^{2}-\bar{\sigma}_{\mathrm{BS}}\left(t_{i}\right)^{2} \cdot t_{i}\right)\right. \\
& \left.-\left(\frac{\bar{\sigma}_{\mathrm{BS}}\left(t_{i+1}\right)}{\gamma_{i+1}}\left(1+\alpha_{i} \Delta t_{i}\right)-\frac{\bar{\sigma}_{\mathrm{BS}}\left(t_{i}\right)}{\gamma_{i}}\right) \cdot \xi\right) .
\end{aligned}
$$

Choosing $\alpha_{i}$ such that

$$
\left(\frac{\bar{\sigma}_{\mathrm{BS}}\left(t_{i+1}\right)}{\gamma_{i+1}}\left(1+\alpha_{i} \Delta t_{i}\right)-\frac{\bar{\sigma}_{\mathrm{BS}}\left(t_{i}\right)}{\gamma_{i}}\right)=0
$$

we have

$$
P\left(t_{i+1} ; t_{i}, \xi\right)=\exp \left(-r \cdot\left(t_{i+1}-t_{i}\right)\right)
$$

Interestingly, the Markov functional model (7)-(8) does necessarily need to be a BlackScholes model having the $\mathbb{Q}^{\mathbb{B}}$-dynamics

$$
\begin{aligned}
\mathrm{d} S(t) & =r S(t) \mathrm{d} t+\sigma_{\mathrm{BS}}(t) \cdot S(t) \cdot \mathrm{d} W^{\mathbb{Q}^{\mathbb{B}}}(t) \\
\mathrm{d} B(t) & =r B(t) \mathrm{d} t,
\end{aligned}
$$

\footnotetext{
${ }^{2}$ Note than in average the interest rate is still $r$.
} 
where $\bar{\sigma}_{\mathrm{BS}}\left(t_{i}\right)^{2}=\frac{1}{t_{i}} \int_{0}^{t_{i}} \sigma_{\mathrm{BS}}(t)^{2} \mathrm{~d} t$. The two models are not the same, although their terminal probability densities (European option prices) and their interest rate dynamics agree. The difference lies in the forward volatility, which may be changed for the Markov functional model through the instantaneous volatility of $x$. Only for $\sigma(t)=\sigma_{\mathrm{BS}}(t)$ we will have the dynamics (9). In this case the $\alpha_{i}$ in (8) will be zero, i.e. we are in the situation of the previous example.

\subsubsection{Calibration to arbitrary Interest Rate Dynamics}

Within the no-arbitrage constraints, it is possible to calibrate the model to a given arbitrary interest rate dynamics by choosing the appropriate drift. To do so, we have to find $\mu\left(t_{i}, \xi\right)$ such that

$$
P\left(t_{i+1} ; t_{i}, \xi\right)=\mathrm{E}^{\mathbb{Q}}\left(\frac{S\left(t_{i}, \xi\right)}{S\left(t_{i+1}, x\left(t_{i+1}\right)\right)} \mid\left\{x\left(t_{i}\right)=\xi-\mu\left(t_{i}, \xi\right) \Delta t_{i}\right\}\right) .
$$

This can be done numerically through a one-dimensional root finder. The functional $S\left(t_{i+1}\right)$ has to be recalibrated in every iteration. ${ }^{3}$

\subsection{Forward Volatility}

The calibration to European option prices (Section 4) and joint movements of asset and interest rates (Section 6.2) still leaves the instantaneous volatility $\sigma$ of the Markovian driver $x$ a free parameter. It may be used to calibrate the forward volatility, i.e. the volatility of an option, conditional we are at time $t>0$ in state $\xi$.

\subsubsection{Example: Black-Scholes Model with a Term Structure of Volatility}

Consider the simple Black-Scholes like example from Section 6.2. For simplicity we consider a Markovian driver without drift, i.e.

$$
x\left(t_{i+1}\right)=x\left(t_{i}\right)+\sigma_{i} \Delta W\left(t_{i}\right) .
$$

together with functionals

$$
S\left(t_{i}, \xi\right)=S(0) \cdot \exp \left(r \cdot t_{i}+\frac{1}{2} \bar{\sigma}_{\mathrm{BS}}\left(t_{i}\right)^{2} t+\frac{\bar{\sigma}_{\mathrm{BS}}\left(t_{i}\right)}{\bar{\sigma}_{i}} \cdot \xi\right)
$$

calibrating to European options with implied volatility $\bar{\sigma}_{\mathrm{BS}}\left(t_{i}\right){ }^{4}$

Then we have that the standard deviation of the increment $x\left(t_{k}\right)-x\left(t_{i}\right)$ is $\left.\bar{\sigma}_{t_{i}, t_{k}} \cdot \sqrt{(} t_{k}-t_{i}\right)$, where $\frac{1}{t_{k}-t_{i}} \sum_{j=i}^{k-1} \sigma_{j}^{2} \Delta t_{j}$. It follows that the implied volatility of an option with maturity $t_{k}$, given we are in state $\left(t_{i}, \xi\right)$, is

$$
\bar{\sigma}_{\mathrm{BS}}\left(t_{k}\right) \cdot \frac{\bar{\sigma}_{t_{i}, t_{k}}}{\bar{\sigma}_{t_{k}}}
$$

Thus a decay in the instantaneous volatility of the driver process will result in a forward volatility decaying with simulation time $t_{i}$ (for fixed maturity $t_{k}$ ).

\footnotetext{
3 The procedure is the same as for done for the calibration of the FX forward within the cross currency Markov functional model, [5].

${ }^{4}$ As before we use the notation $\bar{\sigma}_{i}^{2}:=\frac{1}{t_{i}} \sum_{j=0}^{i-1} \sigma_{j}^{2} \Delta t_{j}$.
} 


\subsubsection{Example: Exponential Decaying Instantaneous Volatility}

Let us consider the case of a time continuous Markovian driver $\mathrm{d} x=\sigma(t) \mathrm{d} W$ with a decaying instantaneous volatility

$$
\sigma(t)=\exp (-a \cdot t), \quad a \neq 0 .
$$

Then we have $\bar{\sigma}_{t_{1}, t_{2}}:=\sqrt{\frac{1}{t_{2}-t_{1}} \int_{t_{1}}^{t_{2}} \sigma(t) \mathrm{d} t}=\sqrt{\frac{-1}{2 a\left(t_{2}-t_{1}\right)}\left(\exp \left(-2 a t_{2}\right)-\exp \left(-2 a t_{1}\right)\right)}$. Assuming functionals

$$
S(t, \xi)=S(0) \cdot \exp \left(r \cdot t+\frac{1}{2} \bar{\sigma}_{\mathrm{BS}}(t)^{2} t+\frac{\bar{\sigma}_{\mathrm{BS}}(t)}{\bar{\sigma}(0, t)} \cdot \xi\right)
$$

we have that the forward volatility for an option with maturity $T$, given we are in $t$, is

$$
\bar{\sigma}_{\mathrm{BS}}(T) \cdot \frac{\bar{\sigma}(t, T)}{\bar{\sigma}(0, T)}=\bar{\sigma}_{\mathrm{BS}}(T) \cdot \sqrt{\frac{T}{T-t} \frac{\exp (-2 a t)-\exp (-2 a T)}{1-\exp (-2 a T)}}
$$

\section{Implementation}

The model may be implemented in the same way as it is done for a one dimensional Markov functional LIBOR model. We do not repeat this here in full detail. For an in depth discussion on the implementation see, e.g., [4, 5, 6, 9]. The basic steps for a numerical implementation are, see Figure 1:

\subsection{Calibration of the Functional Forms}

- Choose a suitable discretization of $(t, x(t))$, i.e. set up a grid $\left(t_{i}, x_{i, j}\right)$.

- For each $x^{*}=x_{i, j}$ :

- Calculate $V^{\text {model }}\left(T, x^{*} ; 0\right)$.

- Find $K^{*}$ such that $V^{\text {market }}\left(T, K^{*} ; 0\right)=V^{\text {model }}\left(T, x^{*} ; 0\right)$.

- Set $S\left(x_{i, j}\right):=K^{*}$. 
Market

Model

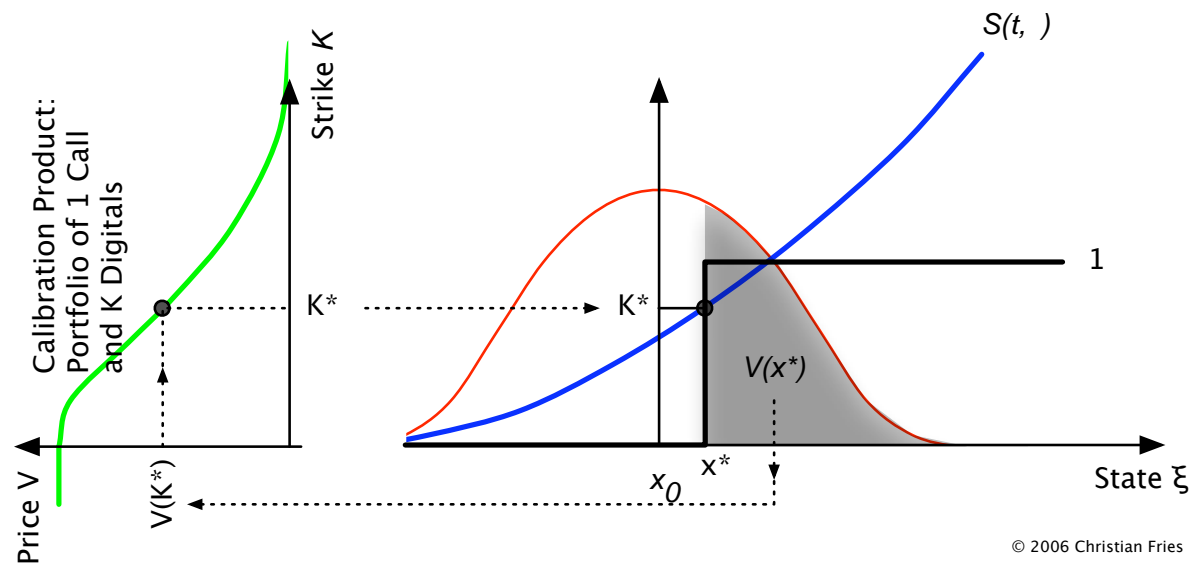

Figure 1: Calibration of the functional $\xi \mapsto S(t, \xi)$ of the Markov functional model for a fixed maturity $t$ : The green curve of prices is given by / inferred from the market. For a given state $x^{*}$ the (model) value $V\left(x^{*}\right)$ of a calibration product (model payout in black) with strike $K^{*}=S\left(t, x^{*}\right)$ is calculated. This can be done without knowing the functional $S(t, \cdot)$, knowing only the distribution of $x(t)$ (red). Looking up the corresponding strike $K^{*}$ (on the market curve (green)) one obtains $K^{*}$. This completes the mapping $x^{*} \mapsto S\left(t, x^{*}\right)=K^{*}$. 


\section{Conclusion}

The equity Markov functional model is an arbitrage free model that

- calibrates to a full two dimensional set of European option prices (parametrized by maturity $T$ and strike $K$ ),

- calibrates to a given joint movement of the asset $S$ and interest rates (as a function of $(t, S))$

- calibrates to (at least) one complex derivative per maturity (means forward volatility sensitive derivatives like a compound or Bermudan option).

The calibration of functionals is given by a very fast inversion, leaving enough calculation reserves for a numerical optimization of the remaining degrees of freedom, i.e. the drift and the instantaneous volatility of the Markovian driver. The calibration of these may be done by induction over time, which significantly reduces the parameters to optimize. 


\section{List of Symbols}

\begin{tabular}{|c|c|}
\hline Symbol & Meaning \\
\hline$x$ & Markovian process (driver). \\
\hline$\sigma$ & Instantaneous volatility of Markovian driver process. \\
\hline $\mathrm{d} W^{\mathbb{Q}}(t)$ & $\mathbb{Q}$ Brownian motion. \\
\hline$S$ & Asset process. \\
\hline $\mathbb{Q}$ & $\begin{array}{l}\text { Equivalent martingal measure with respect to the asset Numéraire } \\
S \text {. }\end{array}$ \\
\hline$(t, \xi) \mapsto S(t, \xi)$ & Functional for the asset $S$ at time $t$ and in state $\{x(t)=\xi\}$. \\
\hline$P(T ; t)$ & Time $t$ value of the zero coupon bond paying 1 at maturity $T$. \\
\hline $\bar{\sigma}_{\mathrm{BS}}(T, K)$ & $\begin{array}{l}\text { Market implied Black-Scholes volatility for a European option with } \\
\text { maturity } T \text { and strike } K \text {. }\end{array}$ \\
\hline$\Phi$ & Cumulative normal distribution function. \\
\hline$V^{\text {model }}\left(T, x^{*} ; 0\right)$ & $\begin{array}{l}\text { Model price of the calibration product with strike at state } x(T)= \\
x^{*} \text {. }\end{array}$ \\
\hline$V^{\text {market }}\left(T, K^{*} ; 0\right)$ & $\begin{array}{l}\text { Market price of the calibration product with strike at asset value } \\
S(T)=K^{*} \text {. }\end{array}$ \\
\hline $\bar{\sigma}_{\mathrm{BS}}(T)$ & $\begin{array}{l}\text { Market implied Black-Scholes volatility for a European option with } \\
\text { maturity } T \text { (considering a Black-Scholes model without smile). }\end{array}$ \\
\hline$\sigma_{\mathrm{BS}}$ & $\begin{array}{l}\text { Instantaneous Black-Scholes volatility. } \\
\bar{\sigma}_{\mathrm{BS}}(T)^{2}=\frac{1}{T} \int_{0}^{T} \sigma_{\mathrm{BS}}(t)^{2} \mathrm{~d} t\end{array}$ \\
\hline
\end{tabular}




\section{References}

[1] Breeden, D. T.; Litzenberger, R. H.: Prices of state-contingent claims implicit in option prices. Journal of Business 51(4), 621?651, 1978.

[2] Brockhaus, Oliver: Implied Monte Carlo. Bachelier Conference Crete, June 2002.

[3] Carr, Peter; Thalela, M.; Zariphopoulou, T.: Closed From option Valuation with Smiles. Working Paper (1999).

[4] Fries, Christian P.: Mathematical Finance. Theory, Modeling, Implementation. Lectures Notes. Frankfurt am Main, 2006,

http://www.christian-fries.de/finmath/book.

[5] Fries, Christian P.; RotT, Marius G.: Cross Currency and Hybrid Markov Functional Models. 2004.

http://www.christian-fries.de/finmath/markovfunctional

[6] Hunt, Phil J.; Kennedy, Joanne E.: Financial Derivatives in Theory and Practice. John Wiley \& Sons, 2000. ISBN 0-471-96717-3.

[7] Hunt, Phil J.; Kennedy, JoAnne E.; Pelsser, Antoon: Markov-Functional Interest Rate Models. Finance and Stochastics, Volume 4 (4), 391-408, 2000. Reprinted in: Hughston, Lane (ed.): The New Interst Rate Models, RISK Publications.

[8] JÄCKEL, PETER: A practical method for the valuation of a variety of hybrid products. Global Derivatives. 2005.

[9] Pelsser, Antoon: Efficient Methods for Valuing Interest Rate Derivatives. Springer Verlag, 2000. ISBN 1-852-33304-9.

\section{Notes}

\section{Suggested Citation}

Fries, Christian P.: Markov Functional Modeling of Equity, Commodity and other Assets. (2006).

http://www.christian-fries.de/finmath/markovfunctionaleqmodel

\section{Updates}

Note that this paper is still work in progress. Please check

http://www.christian-fries.de/finmath/markovfunctionaleqmodel for updates.

\section{Classification}

Classification: MSC-class: 65C05 (Primary), 68U20, 60H35 (Secondary). ACM-class: G.3; I.6.8.

JEL-class: C15, G13.

Keywords: Markov functional model, implied modeling, equity, commodity, lattice model

$$
15 \text { pages. } 1 \text { figures. } 0 \text { tables. }
$$

